\section{Effectiveness of home rehabilitation for ischemic stroke}

\section{Pakaratee Chaiyawat, Kongkiat \\ Kulkantrakorn, Paskorn Sritipsukho}

Faculty of Medicine, Thammasat

University, Pathumtanee, Thailand

\section{Abstract}

The objective of this study was to develop and examine the effectiveness of an individual home rehabilitation program for patients with ischemic stroke. This was a randomized controlled trial in 60 patients with recent middle cerebral artery infarction. After hospital discharge for acute stroke care, they were randomly assigned to receive either a home rehabilitation program for three months (intervention group) or usual care (control group). We collected outcome data over three months after their discharge from the hospital. The Barthel Index (BI), the Modified Rankin Scale (MRS), the health-related quality-of-life index (EQ5D), the Hospital Anxiety and Depression score (HADs), and the Thai Mental State Examination (TMSE) were used to analyze the outcomes. In the intervention group, all outcomes were significantly better $(p<0.05)$ than in the control group, except in the case of TMSE. A favorable outcome, which was defined as minimal or no disability as measured by BI (score $95-100$ ), was achieved by $93.33 \%$ of patients in the intervention group, and 90\% had favorable scores ( 0 or 1$)$ on the MRS. This showed a benefit in reducing disability, with two being the number of patients considered as needed-to-treat (NNT) (95\% CI, 1.0-1.2). All dimensions of EQ-5D in the intervention group were significantly better for quality of life and generic health status than in the control group $(p=0.001)$. Depression was found in one patient (3.33\%) in the intervention group and in two patients $(6.67 \%)$ in the control group. Dementia was found in three patients (10\%) in the intervention group and in four patients $(13.33 \%)$ in the control group. We concluded that an early home rehabilitation program for patients with ischemic stroke in the first threemonth period provides significantly better outcomes in improving function, reducing disability, increasing quality of life, and reducing depression than a program of usual care does.

\section{Introduction}

Stroke is the leading cause of disability in adults around the world, including Thailand. Ischemic stroke caused by large artery atherosclerosis and cardioembolism is found in 20\% and $15 \%$ of stroke patients, respectively. ${ }^{2}$ Individuals who have a stroke also endure a great deal of suffering; for example, physical limitations, emotional trauma, job loss and familial rejection because of the burden. ${ }^{3}$ Most motor and functional recovery usually occurs in the first three months after a stroke. ${ }^{4}$ Moreover, depression and dementia significantly affect the quality of life and physical ability of stroke patients. ${ }^{5.8}$ The duration of hospital stays for rehabilitation of acute stroke patients has decreased, so recovery often is not complete at the time of discharge. ${ }^{9}$ The consequences after discharge may involve not only persistent neurological impairment, but also lifetime disability. An attempt to solve this problem may require a novel rehabilitation program to maximize patients' function in overcoming their disabilities.

Many studies have been conducted to examine rehabilitation of stroke patients, but the results have been inconsistent. Previous studies have documented that patients usually have significant residual physical disability, functional impairment, and reduced quality of life. ${ }^{10}$ Some studies have shown that motor function gradually returns only as a result of spontaneous recovery. Therefore, stroke rehabilitation programs may not improve outcomes. ${ }^{11,12}$

Several studies ${ }^{10,11,13-15}$ have suggested that home rehabilitation is more effective and cheaper than usual care. Rehabilitation begun early in the acute stage optimizes the patient's potential for functional recovery. Early mobilization prevents or minimizes the harmful effects of deconditioning and the potential for secondary impairments. Recovery from stroke and relearning is based on the brain's capacity for reorganization and adaptation. An effective rehabilitation plan capitalizes on this potential and encourages functional use of the involved parts of the body. Activities are selected that are meaningful and important to the patient. Optimal motor learning can be ensured through attention to a number of factors: most importantly, strategy development, feedback, and practice. Rehabilitation can begin as soon as the patient is stabilized medically, typically within 72 hours of insult. Patients may be admitted to a specific stroke unit or neurological unit with rehabilitation services. Evidence supports the benefits of such services in significantly improving functional outcomes when compared to patients not receiving those services.

In most developed countries there is a heavy reliance on hospitals for acute care, whereas home rehabilitation of stroke patients is limited. ${ }^{13,14}$ Because inpatient rehabilitation programs in Thailand are not widely available, the
Correspondence: Kongkiat Kulkantrakorn,

Neurology Division, Department of Internal Medicine, Faculty of Medicine, Thammasat

University, Pathumtanee 12120, Thailand

E-mail: kongkiat1@gmail.com

Key words: effectiveness, home rehabilitation, ischemic stroke

Acknowledgements: this study was supported by funds from Thammasat University. The authors greatly appreciate the cooperation of the physicians and staff at Thammasat University, as well as the caregivers of the patients.

Received for publication: 6 April 2009

Revision received: 29 July 2009.

Accepted for publication: 24 August 2009.

This work is licensed under a Creative Commons Attribution 3.0 License (by-nc 3.0).

(C) Copyright P. Chaiyawat et al., 2009

Licensee PAGEPress, Italy

Neurology International 2009; 1:e10

doi:10.4081/ni.2009.e10

demand for home rehabilitation is increasing. Therefore, a model for effective home rehabilitation for stroke patients will help improve stroke care, and could be applied to other countries. To date, no randomized controlled trial has assessed the effectiveness of a home rehabilitation program for victims of ischemic stroke from middle cerebral artery infarction.

Therefore, our study evaluates the effectiveness of a home rehabilitation program for patients with ischemic stroke. We postulated that the program would be able to improve the activities of daily living (ADL) of stroke patients who had suffered middle cerebral artery infarction, as well as decrease disability, increase quality of life, reduce depression, and alleviate dementia.

\section{Materials and methods}

\section{Design}

This was a randomized controlled trial (RCT). All eligible subjects gave their informed consent, and the study was approved by the Thammasat University Institutional Review Board.

\section{Patient population - inclusion and exclusion criteria}

Patients with stroke as a result of middle cerebral artery infarction were recruited from inpatient wards at Thammasat University Hospital from May 2007 to June 2008. They were screened for eligibility around three days after onset of the stroke. Screening was either 
based on a clinical diagnosis, or was performed exclusively with or aided by magnetic resonance image (MRI) or computer tomography (CT) scanning. The main inclusion criteria of the trial were: stroke from middle cerebral artery infarction, patient's and caregiver's willingness to participate, ability to provide informed consent, and living within 50 miles of the hospital. Patients were excluded if they had uncontrolled hypertension, had severe dysphasia or cognitive impairment, had already been discharged to residential care, had demonstrated previous disability in selfcare, or had been living in a nursing home prior to the stroke.

\section{Randomization}

Eligible patients were stratified by gender and age ( $\leq 40$ years, $>40$ years). Hence, the participants were divided into the following four categories: males aged less than or equal to 40 years, females less than or equal to 40 years, males more than 40 years, and females more than 40 years. In each stratum, each individual was numbered consecutively. These numbers had previously been randomized equally between the intervention group and the control group. After giving informed consent, patients were randomly allocated to receive either a home rehabilitation program (intervention group) or usual care (control group). This method was used to regulate the number of patients who were allocated to the intervention group, which otherwise would have resulted in a significant bias in the study. In addition, block randomization allowed fair allocation of independent persons against the potential bias because of changing practices over the three-month duration of the study, which could have resulted from uneven recruitment between intervention and control groups at the beginning and the end of the study. Randomization was performed by a sequence of sealed envelopes in which the treatment assignment was given, using a random number table and block randomization in a fixed box of each strata.

\section{Intervention group}

Intervention consisted of an individual's home-based exercise program provided by a physical therapist once a month for three months. The physical therapist evaluated a range of functions related to indoor and outdoor mobility, as well as some basic ADL, before providing a home rehabilitation program for the stroke patient. Individual counseling, which focused on education, applying information learned in practical situations, and solving problems occurring at home, was offered to the caregiver if needed. The intervention strategy was based on principles of exercise physiology and motor learning, and had been developed with the combined input of experts, stroke patients, physical therapists, occupational therapists, and speech therapists. It consisted of standardized rehabilitation procedures on an audiovisual CD: passive exercise, active exercise, resistance exercise, and ADL, including putting on and taking off shoes, how to use a cane or wheelchair, etc. The duration and type of therapy were recorded on a case report form by the therapist. Each home program lasted approximately one hour. Patients or caregivers were asked to keep diaries between therapy sessions on the time and type of training. Caregivers were instructed on how to assist patients in ways that allowed patients to use their functional skills as much as possible.

\section{Control group}

Patients in the control group and their family members were given instructions for home rehabilitation prior to discharge from the hospital. The usual care after discharge may include outpatient rehabilitation at the discretion of the patients' physicians. Other treatments were to be recorded in the case report form. The control group did not have follow-up home visits.

\section{Usual care}

1. Patients received services as prescribed by their physicians.

2. All patients had physical therapy when they were admitted to the hospital.

3. Patients and family members were given instruction for home rehabilitation prior to discharge from the hospital.

4. Patients may receive outpatient rehabilitation at the discretion of their physicians.

\section{Outcomes}

The Barthel index (BI) is a weighted scale of 10 items of basic ADL. The possible range of $\mathrm{BI}$ scores is 0 to 100 ; a score equal to or more than 95 is considered as indicative of the ability of independent self-care. ${ }^{15}$

The Modified Rankin Scale (MRS) provides an assessment of the degree of disability. Minor strokes are considered as grades 0 to 2; major strokes are grades 3 to 5 ; while fatalities are grade $6 .^{15}$

Categorizing patients into different outcome groups by the BI or MRS has been used in major clinical trials. ${ }^{15,16}$ This method is an important measure of showing the meaningful benefits of stroke intervention in clinical practice. The health-related quality-of-life index (EQ-5D with five distinct dimensions) concerns mobility, self-care, usual activities, pain/discomfort, and anxiety/depression. The EQ-5D can be informative in describing the dynamics of health-related quality of life during treatment and follow-up. ${ }^{17}$ Depression out- come was assessed by Hospital Anxiety and Depression scores (HADs). Depression was defined as scores equal to or more than 11 (range of scores: 0 to 21). ${ }^{8}$ Dementia outcome was assessed by the Thai Mental State Examination (TMSE). Dementia was defined as scores lower than 23 out of $30 .{ }^{8} \mathrm{EQ}-5 \mathrm{D}$ and HADs have been translated into Thai and validated according to international standards.

\section{Follow-up}

Follow-up visits at patients' residences were scheduled at one, two, and three months after discharge from the hospital. All patients and caregivers were interviewed and evaluated at their residences. Systematic assessments followed a case report from only one assessor. However, a blind study including patient and assessor was not practical for this study. The assessor had adequate training and was accredited in using the National Institute of Health Stroke Scale (NIHSS) and the MRS scale.

\section{Sample size}

This study, with 30 patients per group, had an $80 \%$ probability of achieving a statistically significant result at a two-sided 5\% level if the mean differences between the BI scores for the groups and the standard deviation were 4.8 and 6.7 , respectively. ${ }^{10}$

\section{Statistical analysis}

Data were analyzed by STATA for Windows version 10 software. Descriptive statistics were used to characterize demographics, performance, and clinical characteristics for each group. All analyses were performed on an intention-to-treat (ITT) basis. The continuous outcomes of the BI and utility index were analyzed by analysis of covariance (ANCOVA), with the baseline as a covariate and age and depression as factors in the model. The level of significance was set to 0.05 . Outcomes were classified into one of four categories: minimal or no disability (BI score of 95-100, or MRS score of 0 or 1); moderate disability (BI: 55-94, or MRS: 2 or 3); severe disability (BI: $0-54$, or MRS: 4 or 5); and death. A favorable outcome was defined as minimal or no disability, as measured by scores of 95 to 100 on the BI, and 0 or 1 on the MRS, HADs less than 11, or TMSE equal to or more than $23 .^{17}$

\section{Results}

Sixty-eight subjects were approached and screened for their eligibility to enroll in this study; 60 patients met the inclusion/exclusion criteria (Figure 1). All gave their informed con- 
sent for participation. Eight patients were excluded because of severe aphasia, severe cognitive impairment, living outside the area, or being discharged to residential care. Two patients in the usual care group died because of a cardiovascular problem before the second evaluation at home. There was no significant difference in baseline characteristics and inpatient hospital days between the two groups (Table 1). However, there was a major difference in the proportion of patients with right hemisphere stroke (20\%).

In the intervention group, all outcomes were significantly better $(p<0.05)$, except in the case of TMSE, than in the control group (Table $2)$. At three months, the BI showed more significant improvement in the intervention group than in the usual care group: $96.33 \pm$ 1.04 vs. $66.25 \pm 1.58, p=0.001$ (Figure 2). A favorable outcome, which was defined as minimal or no disability as measured by scores of 95-100 on the BI, was achieved by $93.33 \%$ of patients in the intervention group; $90 \%$ had favorable scores of 0 or 1 on the MRS (Figure 3 ). There was a benefit in reducing disability, with a needed-to-treat (NNT) number of two ( $95 \%$ CI, 1.0 to 1.2 ). The utility index comparison was $0.88 \pm 0.02$ vs. $0.53 \pm 0.02, p=0.06$. In addition, when we compared the mean outcomes for the five dimensions (mobility, selfcare, usual activities, pain/discomfort, anxiety/depression) of the EQ-5D at three months, all dimensions of the EQ-5D in the intervention group were significantly better for quality of life and generic health status than in the control group $(p=0.001)$. HADs were also significantly different between the intervention and control groups. There was an improvement in HADs for both groups, but the intervention group improved more. Depression was found in 20 patients $(66.67 \%)$ at baseline and one $(3.33 \%)$ patient at three months in the intervention group; while it was found in 25 (83.33\%) patients and two (6.67\%) patients, respectively, in the control group. TMSE scores were not significantly different between the intervention group and the control group. At three months, dementia was found in three patients (10\%) in the intervention group and in four patients (13.33\%) in the control group. There were no serious adverse events in either group. Compliance with the intervention, based on daily records, was $95 \%$.

\section{Discussion}

The present study has provided additional data supporting the remarkable benefits of a home rehabilitation program. After three months, home rehabilitation intervention produced greater gains and higher rates of functional independence, ability, and quality of life

Table 1. Subject characteristics and baseline measures.

\begin{tabular}{|c|c|c|}
\hline & $\begin{array}{l}\text { Intervention Group } \\
\qquad(\mathrm{n}=30)\end{array}$ & $\begin{array}{c}\text { Control Group } \\
(\mathrm{n}=30)\end{array}$ \\
\hline Age (yrs), mean (SD) & $67(10)$ & $66(11)$ \\
\hline Male, $n(\%)$ & $14(47)$ & $13(43)$ \\
\hline BMI, mean (SD) & $24.8(1.6)$ & $24.6(2.4)$ \\
\hline Elementary education, $\mathrm{n}(\%)$ & $28(93)$ & $28(93)$ \\
\hline $\begin{array}{l}\text { Length of stay in hospital before } \\
\text { discharge, mean (SD) }\end{array}$ & $10(1.7)$ & $10.9(2.3)$ \\
\hline Right hemisphere stroke, n (\%) & $18(60)$ & $12(40)$ \\
\hline $\begin{array}{l}\text { Medical history } \\
\text { Hypertension } \\
\text { Diabetes } \\
\text { High cholesterol } \\
\text { Atrial fibrillation/Ischemic } \\
\text { heart disease }\end{array}$ & $\begin{array}{l}17(57) \\
16(53) \\
8(27) \\
7(23)\end{array}$ & $\begin{array}{l}17(57) \\
18(60) \\
6(20) \\
8(27)\end{array}$ \\
\hline $\begin{array}{l}\text { National Institute of Health Stroke } \\
\text { Scale (NIHSS), mean (SD) }\end{array}$ & $16.4(4.1)$ & $17.8(3.9)$ \\
\hline Thai Mental State Examination (TMSE), mean (SD) & $24.4(2.0)$ & $23.8(1.9)$ \\
\hline Hospital Anxiety and Depression Scale (HADs), mean (SD) & $16.1(7.6)$ & $16.4(4.9)$ \\
\hline Barthel Index, mean (SD) & $31.7(5.9)$ & $33.2(4.8)$ \\
\hline $\begin{array}{l}\text { Modified Rankin scale, n (\%): } \\
\text { Minor strokes (Grades 0-2) } \\
\text { Major strokes (Grades 3-5) }\end{array}$ & $\begin{array}{c}0 \\
30(100)\end{array}$ & $\begin{array}{c}0 \\
30(100)\end{array}$ \\
\hline Utility index, mean (SD) & $-0.14(0.08)$ & $-0.11(0.13)$ \\
\hline
\end{tabular}

* $p$ by independent sample t-test and Fisher's exact test, significant at $p<0.05$.

Table 2. Results of ANCOVA adjusted for age, depression, dementia, and baseline measurement of outcome at three months.

\begin{tabular}{|c|c|c|c|}
\hline In & $\begin{array}{l}\text { rvention group } \\
(\mathrm{n}=30)\end{array}$ & $\begin{array}{l}\text { Control group } \\
(\mathrm{n}=28)\end{array}$ & $\begin{array}{c}\text { Mean difference } \\
95 \% \mathrm{CI}\end{array}$ \\
\hline Barthel index, mean (SE) & $96.3(1.0)$ & $66.3(1.6)$ & $\begin{array}{c}56.32 \\
(25.59,87.04)\end{array}$ \\
\hline $\begin{array}{l}\text { Modified Rankin scale, n (\%) } \\
\text { Minor strokes (Grades 0-2) } \\
\text { Major strokes (Grades 3-5) }\end{array}$ & $\begin{array}{c}28(93.33) \\
2(6.66)\end{array}$ & $\begin{array}{c}3(10) \\
25(83.33)\end{array}$ & \\
\hline Utility index, mean (SE) & $0.88(0.02)$ & $0.53(0.02)$ & $\begin{array}{c}0.57 \\
(0.17,0.97)\end{array}$ \\
\hline $\begin{array}{l}\text { Thai Mental State Examination } \\
\text { (TMSE), mean (SE) }\end{array}$ & $24.63(0.35)$ & $24.07(0.33)$ & $\begin{array}{c}1.97 \\
(-0.12,4.05)\end{array}$ \\
\hline $\begin{array}{l}\text { Hospital Anxiety and Depression } \\
\text { Scale (HADs), mean (SE) }\end{array}$ & $5.1(0.72)$ & $9.07(0.32)$ & $\begin{array}{c}15.33 \\
(2.88,27.79)\end{array}$ \\
\hline
\end{tabular}

than did usual care. Intervention appears to have accelerated recovery within three months compared to usual care. The factors responsible for this superiority of organized stroke rehabilitation may include support from the family system, the initiation of earlier rehabilitation, the home environment of rehabilitation, an individually tailored program with audiovisual materials, and close followup..$^{10,12,14,18}$ An assessor evaluated each patient on four separate occasions, and a physical therapist provided three home rehabilitation visits all of which contributed to reducing nonresponse. The patients received the physical therapist's telephone number for consultation about the home rehabilitation program. Compliance with the intervention (as indicated by daily records) at one, two, and three months was $94 \%, 95 \%$, and $95 \%$, respectively. The response rate achieved was $100 \%$. The success of rehabilitation also depends on personal factors of stroke patients, such as age, education, socioeconomic status, medical history, and family relationships. ${ }^{10,19}$ Moreover, audiovisual materials (video CDs) may aid in recovery, which could be explained by the mirror neuron theory. Buccino et al. (2006) ${ }^{20}$ proposed the mirror neuron theory in the recovery of motor function and the reorganization of neural network integration, involving both the 
motor and sensory systems. Motor imitation is a complex cognitive function that incorporates several stages, including motor observation (i.e., visual perception of ecologically valid movements), motor imagery, and motor execution. Moreover it has been suggested that motor imagery might be beneficial to the recovery of motor functions after a stroke.

Most patients and caregivers prefer home rehabilitation, because of the opportunity to be closer to their families. This might reflect the strong bonds of the extended family system, which could improve their quality of life. ${ }^{19}$ Past studies have shown that rehabilitation intervention for stroke patients in the early phase of recovery appears to accelerate gains in function and quality of life ${ }^{21}$ Additionally, wellorganized rehabilitation services initiated immediately after a stroke can improve longterm outcomes. Prior studies have demonstrated an improvement of function based on six to eight hours/day of constraint-induced exercise. $^{22}$ On the contrary, our protocol had one hour/day only, with encouragement to practice independently. Additionally, the availability of audiovisual material is helpful as a resource for an intensive, motivated, and progressive program..$^{23}$

Neuropsychiatric disorders such as depression and dementia after a stroke become increasingly important in stroke survivors. ${ }^{24}$ The interaction between depression and neurological disease is complex. When depression precedes the onset of neurological disease, it is often unclear whether depression is the first manifestation of the illness or coincidentally preceded the onset of an ensuring brain disease. Previous studies on the prevalence of depression suggest that it varies widely, ranging from $7-76 \%{ }^{25}$ In Thailand, a one-year cumulative incidence of depression after a stroke was reported in $12 \%$ of patients..$^{26}$ In our study, at three months depression was found in one patient $(3.33 \%)$ in the intervention group, and in two patients (6.67\%) in the control group. This variation perhaps is due to inconsistent methodology. ${ }^{25}$ In our study, there was an improvement in the Hospital Anxiety and Depression scores (HADs) in both groups, but the intervention group improved more. The reason for this is not yet clear. The psychological support from home visits and family surroundings may have helped reduce depression in the intervention group more than in the control group. A previous study has reported a correlation between improved quality of life, improved self-care ability, and decreased depression. In our study, depression is associated also with low health-related quality of life.

A considerable decline in cognitive function, which can occur shortly after a stroke, has been reported by several studies. ${ }^{27,28}$ In a recent study, about $30 \%$ of stroke patients developed dementia; stroke increased the risk of demen-

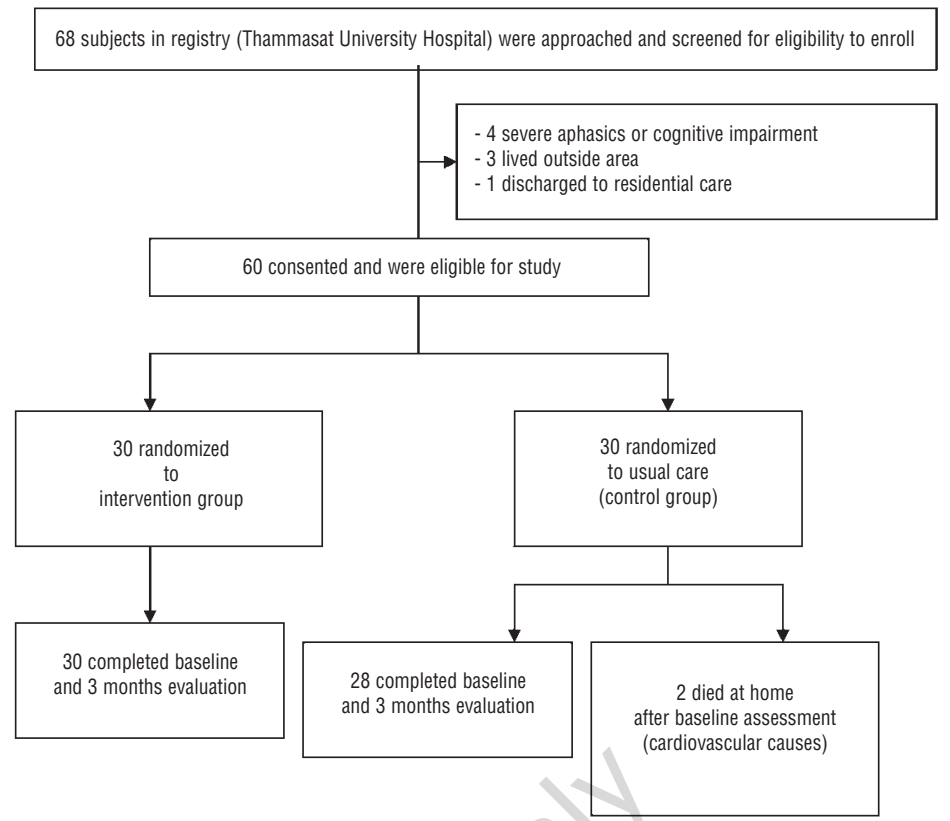

Figure 1. Study flow chart.

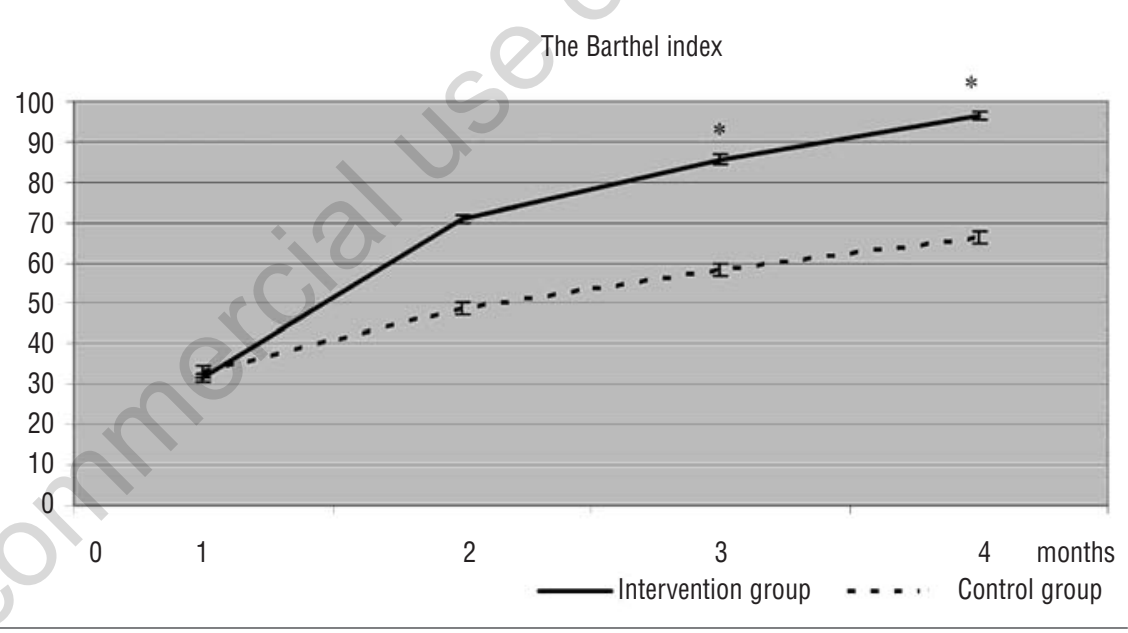

Figure 2. Post-treatment effects of the intervention for three months, adjusted for age, depression, and baseline measurement of outcome by the Barthel index. ${ }^{*} p=0.001$ (ANCOVA, significant at $p<0.05$ ).

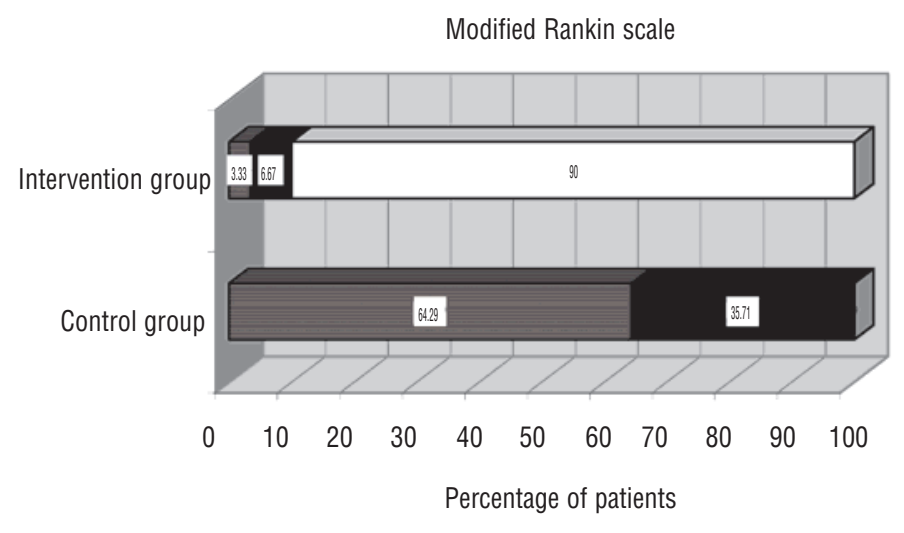

Severe disability (4 or 5) $\quad$ Moderate disability (2 or 3) $\square$ Minimum or no disability (0 or 1)

Figure 3. Outcome of the modified rankin scale at three months, according to treatment group.

[Neurology International 2009; 1:e10]

[page 39] 
tia by 4-12 times. In our study, TMSE was not significantly different between the control group and the intervention group. The reason for this is not yet clear. Dementia might have commenced before the stroke. Barba et al. $(2000)^{29}$ found a prevalence of dementia in $11 \%$ of the elderly; but this study could have been for too short of a term for a thorough analysis. However, our study also has some limitations. It was an efficacy study targeted at ischemic stroke from middle cerebral artery infarction, and so the result may not be applicable to all stroke rehabilitation. In addition, patients with severe stroke were excluded. The evaluation and intervention was done after patients had been discharged from the hospital; therefore, each evaluation time point was not measured directly from the stroke onset. However, the average inpatient stay for each group was approximately 10 days after acute stroke. Thus, the results are still applicable to the time point in this study.

Our study demonstrated that early home rehabilitation service during the first threemonth period in patients with ischemic stroke provides a better outcome for improving function and reducing disability compared to the usual rehabilitation practice.

The implementation of this program will certainly enhance stroke survivors' recovery and quality of life. Further studies on the longterm outcomes and cost-effectiveness of this intervention should be explored, however.

\section{References}

1. Viriyavejakul A, Senanarong V, Prayoonwiwat $\mathrm{N}$, et al. Epidemiology of stroke in the elderly in Thailand. J Med Assoc Thai 1998;81:497-505.

2. Dharmasaroja P. Baseline characteristics of patients with acute ischemic stroke in a suburban area of Thailand. J Stroke Cerebrovasc Dis 2008; 17:82-5.

3. American Heart Association. Heart Disease and Stroke Statistics - 2004 Update. Dallas, Texas: American Heart Association; 2003.

4. Brandstater ME. An overview of stroke rehabilitation. Stroke 1990;21:1140-2.

5. Burvill PW, Johnson GA, Jamrozik KD, et al. Anxiety disorders after stroke: results from the Perth Community Stroke Study.
Br J Psychiatry 1995;166:328-32.

6. Page SJ, Levine P, Sisto SA, et al. Mental practice combined with physical practice for upper-limb motor deficit in subacute stroke. Phys Ther 2001;81:1455-62.

7. Robinson-Smith G, Johnston MV, Allen J. Self-care self-efficacy, quality of life, and depression after stroke. Arch Phys Med Rehabil 2000;81:460-4.

8. Stephens S, Kenny RA, Rowan E, et al. Neuropsychological characteristics of mild vascular cognitive impairment and dementia after stroke. Int $\mathrm{J}$ Geriatr Psychiatry 2004;19:1053-7.

9. Dam M, Tonin P, Casson S, et al. The effects of long-term rehabilitation therapy on poststroke hemiplegic patients. Stroke 1993;24:1186-91.

10. Studenski S, Duncan PW, Perera S, et al. Daily functioning and quality of life in a randomized controlled trial of therapeutic exercise for subacute stroke survivors. Stroke 2005;36:1764-70.

11. Duncan P, Richards L, Wallace D, et al. A randomized, controlled pilot study of a home-based exercise program for individuals with mild and moderate stroke. Stroke 1998;29:2055-60.

12. Feys HM, De Weerdt WJ, Selz BE, et al. Effect of a therapeutic intervention for the hemiplegic upper limb in the acute phase after stroke. A single-blind, randomized, controlled multicenter trial. Stroke 1998; 29:785-92.

13. Anderson CS, Jamrozik KD, StewartWynne EG. Patterns of acute hospital care, rehabilitation, and discharge disposition after acute stroke: the Perth Community Stroke study 1989-1990. Cerebrovasc Dis 1994;4:344-53.

14. Young J. Is stroke better managed in the community? Br Med J 1994;309:1356-8.

15. Duncan PW, Jorgensen HS, Wade DT. Outcome measures in acute stroke trials: a systemic review and some recommendations to improve practice. Stroke 2000;31: 1429-38.

16. Kwiatkowski TG, Libman RB, Frankel M, et al. Effects of tissue plasminogen activator for acute ischemic stroke at one year. $\mathrm{N}$ Engl J Med 1999;340:1781-7.

17. Brooks R, Rabin R, de Charro F. The measurement and valuation of health status using EQ-5D: A European perspective (Evidence from the EuroQol BIOMED research programme). London: Kluwer Academic Publishers; 2005.

18. Anderson C, Rubenach S, Mhurchu CN, et al. Home or hospital for stroke rehabilitation? Results of a randomized controlled trial: I: Health outcomes at 6 months. Stroke 2000;31:1024-31.

19. Heinemann AW, Roth EJ, Cichowski K, et al. Multivariate analysis of improvement and outcome following stroke rehabilitation. Arch Neurol 1987;44:1167-72.

20. Buccino G, Solodkin A, Small SL. Functions of the mirror neuron system: Implications for neurorehabilitation. Cog Behav Neurol 2006;19:55-63.

21. Stroke Unit Trialists' Collaboration. Organised inpatient (stroke unit) care for stroke (Cochrane Review). In: The Cochrane Library, Issue 2. Chichester, UK: John Wiley \& Sons, 2004.

22. Liepert JH, Bauder H, Wolfgang HR, et al. Treatment-induced cortical reorganization after stroke in humans. Stroke 2003;31: 1210-6.

23. Shafer DW, Van der Loos HFM. Integrated video and computerized functional assessment. Proceedings RESNA 1995, 146-148, Vancouver, BC, Canada; 1995.

24. Bourgeois JA, Hilty DM, Chang CH, et al. Poststroke neuropsychiatric illness: and integrated approach to diagnosis and management. Curr Treat Options Neurol 2004; 6:403-20.

25. Veazey C, Aki SO, Cook KF, et al. Prevalence and treatment of depression in Parkinson's disease. J Neuropsychiatry Clin Neurosci 2005;17:10-23.

26. Kulkantrakorn K, Jirapramukpitak T. A prospective study in one year cumulative incidence of depression after ischemic stroke and Parkinson's disease: A preliminary study. J Neurol Sci 2007;263:165-8.

27. Tham W, Auchus AP, Thong M, et al. Progression of cognitive impairment after stroke: one year results from a longitudinal study of Singaporean stroke patients. J Neurol Sci 2002;203-204:49-52.

28. Sachdev PS, Brodaty H, Valenzuela MJ, et al. Progression of cognitive impairment in stroke patients. Neurology 2004;63:161823.

29. Barba R, Martinez-Espinosa S, RodriguezGarcia E, et al. Poststroke dementia: Clinical features and risk factors. Stroke 2000;31:1494-501. 